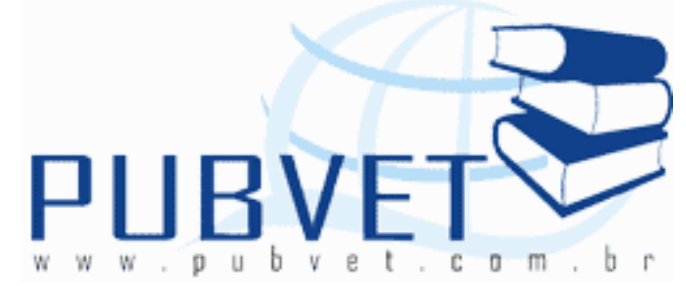

PUBVET, Publicações em Medicina Veterinária e Zootecnia.

\title{
Utilização de dois métodos de classificação andrológica por pontos em touros jovens da raça Braford
}

Flávio Guiselli Lopes ${ }^{1}$, Celso Koetz Junior ${ }^{2}$, Flávio Antônio Barca Junior ${ }^{3}$, Luis Afonso Marques Claus ${ }^{3}$, Silvio Renato Oliveira Menegassi ${ }^{4}$, Luigi Carrer Filho ${ }^{5}$

${ }^{1}$ Médico Veterinário, Doutor em Reprodução e Produção Animal, professor do Curso de Medicina Veterinária da Universidade Norte do Paraná (UNOPAR). Autor para contato, e-mail: lopesufv@yahoo.com.br

${ }^{2}$ Médico Veterinário, Doutorando em Ciência Animal pela Universidade Estadual de Londrina, professor do Curso de Medicina Veterinária da Universidade Norte do Paraná (UNOPAR).

${ }^{3}$ Médico Veterinário, Doutorando em Ciência Animal pela Universidade Estadual de Londrina (UEL).

${ }^{4}$ Médico Veterinário, Doutorando em Zootecnia pela Universidade Federal do Rio Grande do Sul (UFRGS).

${ }^{5}$ Médico Veterinário, Mestrando em Saúde e Produção de Ruminantes pela Universidade Norte do Paraná (UNOPAR).

\section{Resumo}

O objetivo do presente estudo foi realizar o exame andrológico completo e estudar a relação entre dois métodos de classificação andrológica por pontos em touros jovens da raça Braford. Cento e quarenta e três touros jovens da raça Braford, hígidos, com peso médio de $521,48 \pm 47,06 \mathrm{Kg}$ e idade média de 
LOPES, F.G. et al. Utilização de dois métodos de classificação andrológica por pontos em touros jovens da raça Braford. PUBVET, Londrina, V. 7, N. 12, Ed. 235, Art. 1551, Junho, 2013.

18 meses, foram submetidos ao exame andrológico completo. Posteriormente, os touros foram pontuados em dois métodos de classificação andrológica, o BSE e o CAP. O valor médio observado para perímetro escrotal foi de $35,85 \pm$ $2,28 \mathrm{~cm}$. A média observada para turbilhonamento, vigor espermático e motilidade progressiva retilínea foi de 4,01 $\pm 0,84,4,06 \pm 0,82$ e 80,29 \pm $8,31 \%$, respectivamente. Quanto às características morfológicas, foi observada média de 3,42 $\pm 5,29,3,41 \pm 4,64,6,83 \pm 9,04 \%$, para os defeitos maiores, defeitos menores e defeitos espermáticos totais, respectivamente. Com relação aos dois métodos de classificação andrológica, o BSE e o CAP obtiveram pontuação média de 83,69 $\pm 11,84$ (satisfatório) e 93,30 \pm 7,81 (excelente), respectivamente. As estimativas de correlações simples de Pearson entre dois métodos de classificação andrológica (BSE e CAP) e o perímetro escrotal e, entre as características físicas do sêmen, foram consideradas positivas e significativas, já, para as características morfológicas do sêmen, as correlações foram consideradas negativas e significativas. Quando foi correlacionado o BSE e o CAP observou-se correlação positiva e significativa.

Palavras-chave: Andrologia, Touro, Sêmen

\title{
Two methods of breeding soundness evaluation used in the evaluation of young of braford breed
}

\begin{abstract}
The aim of this study was to do a complete breeding soundness examination and study the relationships between two breeding soundness classification methods in young Braford bulls. One hundred and forty three young and healthy Braford bulls, with an average weight of 521.48 ( $\pm 47.06 \mathrm{~kg}$ ) and average age of 18 months underwent complete breeding soundness examinations. Thereafter the bulls were scored using two breeding soundness methods, the BSE (Breeding Soundness Evaluation) and the CAP (classificação andrológica por pontos). The average value observed for scrotal circumference was $35.85( \pm 2.28 \mathrm{~cm})$. The average observed for turbulence was 4.01 ( \pm
\end{abstract}


LOPES, F.G. et al. Utilização de dois métodos de classificação andrológica por pontos em touros jovens da raça Braford. PUBVET, Londrina, V. 7, N. 12, Ed. 235, Art. 1551, Junho, 2013.

0.84 ), for progressive motility was 4.06 ( \pm 0.82 ) and for rectilinear sperm vigor was 80.29 ( $\pm 8.31 \%)$. As for the morphological features, an average of 3.42 ( \pm 5.29 ) was observed for major defects, 3.41 ( \pm 4.64$)$ for minor defects and $6.83 \pm(9.04 \%)$ for total sperm defects. Regarding the two breeding soundness methods, BSE gave a mean score of 83.69 ( \pm 11.84 ) (satisfactory) and the CAP gave a mean score of 93.30 ( \pm 7.81) (excellent). Estimates of simple Pearson correlations between the two breeding soundness methods (BSE and CAP) and scrotal perimeter, as well as the physical characteristics of the semen were considered positive and significant. The reason is that for the morphological characteristics of sperm the correlations were negative and significant. When BSE correlated with CAP there was a significant positive correlation.

Keywords: Andrology, Taurus, Semen.

\section{INTRODUÇÃO}

A seleção de reprodutores por meio do exame andrológico tem por finalidade a obtenção de informações que permitem estimar o potencial reprodutivo dos touros (Silva, 2011).

Um dos fatores determinantes da eficiência reprodutiva dos rebanhos de corte é a fertilidade dos touros. A utilização inadequada do potencial reprodutivo dos machos, leva ao aumento da demanda de reprodutores, ocasionando a utilização de touros sem qualquer seleção genética, quer para as características morfológicas, quer para as funcionais, como fertilidade e precocidade sexual (Fonseca et al., 1991).

Vários estudos têm sido realizados no sentido de estabelecer critérios e parâmetros para avaliar o potencial individual dos touros. Segundo Vale Filho (1997), muitas escolas clássicas influenciaram no processo de avaliação, diagnóstico e classificação de touros no Brasil.

Entre os padrões de avaliação, Vale Filho et al. (1999) propõem o uso da classificação andrológica por pontos (CAP), sendo estabelecido um índice de 0 a 100 pontos para comparação entre touros clinicamente normais sob o ponto 
LOPES, F.G. et al. Utilização de dois métodos de classificação andrológica por pontos em touros jovens da raça Braford. PUBVET, Londrina, V. 7, N. 12, Ed. 235, Art. 1551, Junho, 2013.

de vista andrológico.

O CAP constitui-se um importante índice de confronto entre touros de uma mesma raça ou de comparações entre raças criadas num mesmo ambiente. Além disso, somente touros normais devem ser classificados pelo CAP, havendo sempre, em cada rebanho, o ranqueamento dos reprodutores, onde os de maior pontuação devem ter maior número de matrizes desafiadas (Vale Filho et al., 2001).

Atualmente, o CBRA (1998) preconiza duas tabelas de pontuação. Para avaliação da capacidade reprodutiva de touros europeus (Bos taurus taurus), o mais difundido tem sido o padrão norte-americano "Breeding Soundness Evaluation" (BSE), proposto por Chenoweth \& Ball (1980).

O BSE avalia e pontua os reprodutores de acordo com o perímetro escrotal em função da faixa etária, conjuntamente com os aspectos físicos (motilidade espermática progressiva retilínea e vigor espermático) e morfológicos (defeitos maiores e defeitos totais) do sêmen, sendo pontuados no máximo em 40, 20 e 40 pontos, respectivamente (Chenoweth \& Ball, 1980).

Fonseca et al. (1997), com a finalidade de obter uma tabela de simples aplicação que pudesse predizer a fertilidade de touros Bos taurus indicus, propuseram uma tabela específica para avaliação andrológica de reprodutores de origem zebuína.

De forma similar ao padrão norte americano "BSE", o método apresentado por Fonseca et al. (1997), propõe uma nova classificação ao perímetro escrotal de acordo com a faixa etária e, também, propõe modificações na pontuação quanto aos aspectos físicos (motilidade espermática progressiva retilínea e vigor espermático) e morfológicos (defeitos maiores e defeitos totais) do sêmen, que são pontuados no máximo em 25 e 35 pontos, respectivamente.

O objetivo do presente estudo foi realizar o exame andrológico completo e estudar a relação entre dois métodos de classificação andrológica por pontos em touros jovens da raça Braford. 
LOPES, F.G. et al. Utilização de dois métodos de classificação andrológica por pontos em touros jovens da raça Braford. PUBVET, Londrina, V. 7, N. 12, Ed. 235, Art. 1551, Junho, 2013.

\section{MATERIAL E MÉTODOS}

O presente estudo foi realizado em propriedade rural, no município de Naviraí, região centro-oeste do estado do Mato Grosso do Sul, situada na latitude $23^{\circ} 03^{\prime} 54^{\prime \prime}$ sul e longitude 54011'26" oeste de Greenwich, a 362 metros de altitude, com temperatura média anual de $22^{\circ} \mathrm{C}$ e clima tropical.

Cento e quarenta e três touros jovens da raça Braford, hígidos, com peso médio de 521,48 $\pm 47,06 \mathrm{Kg}$ e idade média de 18 meses, foram submetidos ao exame andrológico completo (mensuração do perímetro escrotal e avaliação morfológica do sêmen e das características físicas).

Os touros foram criados em pastagem de Brachiaria brizantha, com rotação de piquetes e alimentação suplementar. Água e o sal mineral foram fornecidos ad libitum durante todo o período.

Primeiramente, para o exame andrológico, os touros foram contidos individualmente em tronco de contenção apropriado, sendo realizada a mensuração do perímetro escrotal, na região mais larga do saco escrotal (Vasconcelos et. al., 2003).

Para obtenção dos ejaculados, foi utilizado o método do eletroejaculação. O sêmen coletado dos reprodutores foi avaliado conforme as recomendações do Colégio Brasileiro de Reprodução Animal (CBRA 1998).

As avaliações físicas do sêmen (turbilhonamento, vigor espermático motilidade progressiva retilínea) foram realizadas na própria fazenda e, as avaliações morfológicas do sêmen (defeitos maiores, menores e totais), no laboratório próprio do Médico Veterinário.

Para a avaliação do turbilhonamento (movimento em massa dos espermatozóides), foi utilizado escala de 0 a 5 com auxílio de microscópio binocular convencional (aumento de $100 \mathrm{x}$ ), colocando uma gota de sêmen sobre uma lâmina previamente aquecida (temperatura de $37^{\circ} \mathrm{C}$ ).

Em seguida, com outra gota de sêmen, entre lâmina e lamínula previamente aquecida (temperatura de $37^{\circ} \mathrm{C}$ ), foi avaliado a motilidade espermática progressiva retilínea (percentual de espermatozóides com movimento) e o vigor espermático (intensidade do movimento dos 
LOPES, F.G. et al. Utilização de dois métodos de classificação andrológica por pontos em touros jovens da raça Braford. PUBVET, Londrina, V. 7, N. 12, Ed. 235, Art. 1551, Junho, 2013.

espermatozóides), numa escala de 0 a $100 \%$ e de 0 a 5, respectivamente, com auxílio de um microscópio binocular convencional (aumento de $400 \mathrm{x}$ ).

Para avaliação das características morfológicas, uma quantidade de sêmen $(100 \mu \mathrm{l})$, de cada ejaculado, foi armazenada em tubo de plástico, contendo $1,0 \mathrm{~mL}$ de solução formol salina tamponada. Para esta avaliação, foi utilizada a classificação descrita por Blom (1983) e adotada pelo CBRA (1998), sendo contados 200 espermatozóides, em esfregaço corado, com auxílio de um microscópio binocular convencional (aumento de $1000 \mathrm{x}$ ).

Após o exame andrológico completo, os touros foram pontuados em dois métodos de classificação andrológica, o BSE e o CAP.

O método BSE classifica os reprodutores de acordo com perímetro escrotal em função da faixa etária, conjuntamente com os aspectos físicos (motilidade espermática progressiva retilínea e vigor espermático) e morfológicos (defeitos maiores e defeitos totais) do sêmen, sendo pontuados no máximo em 40, 20 e 40 pontos, respectivamente (Chenoweth \& Ball, 1980).

Depois de pontuados os touros foram enquadrados em três categorias: de 60 a 100 pontos (satisfatório), de 30 a 59 pontos (questionável) e de 30 a 0 ponto (insatisfatório) (Chenoweth \& Ball, 1980) (Tabela 1).

Já, o método CAP, proposto por Fonseca et al. (1997), dá uma nova classificação ao perímetro escrotal de acordo com a faixa etária, valendo até 40 pontos, no máximo e, também, propõe modificações na pontuação quanto aos aspectos físicos (motilidade espermática progressiva retilínea e vigor espermático) e morfológicos (defeitos maiores e defeitos totais) do sêmen, que são pontuados no máximo em 25 e 35 pontos, respectivamente.

Em seguida, os touros foram pontuados e enquadrados em quatro categorias: de 86 a 100 pontos (excelente), de 66 a 86 pontos (muito bom), de 40 a 66 (bom) e de 40 a 0 ponto (regular) (Fonseca et al., 1997) (Tabela 2).

Foi realizada a análise descritiva das características estudadas (média, desvio-padrão, coeficiente de variação e amplitude). Além disso, foi calculada, 
LOPES, F.G. et al. Utilização de dois métodos de classificação andrológica por pontos em touros jovens da raça Braford. PUBVET, Londrina, V. 7, N. 12, Ed. 235, Art. 1551, Junho, 2013.

a correlação simples de Pearson de dois métodos de classificação andrológica (BSE e CAP) em relação às características estudadas, utilizando o pacote estatístico Minitab 13.0 (State College, PA, USA: Minitab Inc. 2000).

Tabela 1. Método BSE, sugerida para classificação de touros da subespécie Bos taurus taurus, baseada no perímetro escrotal e nas características físicas e morfológicas do sêmen.

\begin{tabular}{|c|c|c|c|c|}
\hline \multirow[t]{2}{*}{ Parâmetro } & \multicolumn{4}{|c|}{ Classificação } \\
\hline & Excelente & Bom & Regular & Fraco \\
\hline \multicolumn{5}{|l|}{ Características físicas } \\
\hline Vigor (0-5) & 5 & $4-5$ & 4 & $<3$ \\
\hline Motilidade progressiva (\%) & $>70$ & $60<70$ & $50<60$ & $<50$ \\
\hline Pontos outorgados & 20 & 12 & 10 & 3 \\
\hline \multicolumn{5}{|l|}{ Características morfológicas } \\
\hline Defeitos maiores (\%) & $<10$ & $10<19$ & $20<29$ & $>29$ \\
\hline Defeitos totais (\%) & $<25$ & $26<39$ & $40<59$ & $>59$ \\
\hline Pontos outorgados & 40 & 25 & 10 & 3 \\
\hline \multicolumn{5}{|l|}{ Perímetro escrotal (cm) } \\
\hline \multicolumn{5}{|l|}{ Idade em meses } \\
\hline 09 a 11 & $>30,0$ & $26,0<30,0$ & $<26,0$ & $<26,0$ \\
\hline 12 a 14 & $>34,0$ & $30,0<34,0$ & $<30,0$ & $<30,0$ \\
\hline 15 a 20 & $>36,0$ & $31,0<36,0$ & $<31,0$ & $<31,0$ \\
\hline 21 a 30 & $>38,0$ & $32,0<38,0$ & $<32,0$ & $<32,0$ \\
\hline$>30$ & $>39,0$ & $34,0<39,0$ & $<34,0$ & $<34,0$ \\
\hline Pontos outorgados & 40 & 24 & 10 & 10 \\
\hline Total de pontos do reprodut & \multicolumn{4}{|c|}{ Interpretação } \\
\hline $60-100$ pontos & \multicolumn{3}{|c|}{ Touro Satisfatório } & \\
\hline $30-59$ pontos & \multicolumn{3}{|c|}{ Touro Questionável } & \\
\hline$<30$ pontos & \multicolumn{3}{|c|}{ Touro Insatisfatório } & \\
\hline
\end{tabular}

Fonte: Chenoweth \& Ball (1980). 
LOPES, F.G. et al. Utilização de dois métodos de classificação andrológica por pontos em touros jovens da raça Braford. PUBVET, Londrina, V. 7, N. 12, Ed. 235, Art. 1551, Junho, 2013.

Tabela 2. Método CAP, sugerida para classificação de touros da subespécie Bos taurus indicus, baseada no perímetro escrotal e nas características físicas e morfológicas do sêmen.

\begin{tabular}{|c|c|c|c|c|}
\hline \multirow[t]{2}{*}{ Parâmetro } & \multicolumn{4}{|c|}{ Classificação } \\
\hline & Excelente & Muito Bom & Bom & Questionável \\
\hline \multicolumn{5}{|l|}{ Características físicas } \\
\hline Vigor $(0-5)$ & 5 & $4<5$ & $3<4$ & $<3$ \\
\hline Motilidade progressiva (\%) & $>75$ & $60-75$ & $30<60$ & $<30$ \\
\hline Pontos outorgados & $21<25$ & $16<21$ & $10<16$ & $<10$ \\
\hline \multicolumn{5}{|l|}{ Características morfológicas } \\
\hline Defeitos maiores (\%) & $>5$ & $5-10$ & $10-20$ & $>20$ \\
\hline Defeitos totais (\%) & $>10$ & $10-15$ & $15-30$ & $>30$ \\
\hline Pontos outorgados & $30-35$ & $25<30$ & $15<25$ & $<15$ \\
\hline \multicolumn{5}{|l|}{ Perímetro escrotal $(\mathrm{cm})$} \\
\hline \multicolumn{5}{|l|}{ Idade em meses } \\
\hline 07 a 12 & 21,0 & $19,5<21,0$ & $17,5<19,5$ & $<17,5$ \\
\hline 12 a 18 & 26,0 & $24,0<26,0$ & $21,5<24,0$ & $<21,5$ \\
\hline 18 a 24 & 31,5 & $28,5<31,5$ & $26,0<28,5$ & $<26,0$ \\
\hline 24 a 36 & 35,0 & $32,0<35,0$ & $29,0<32,0$ & $<29,0$ \\
\hline 36 a 48 & 37,0 & $33,5<37,0$ & $30,5<33,5$ & $<30,5$ \\
\hline$>48$ & 39,0 & $36,0<39,0$ & $33,0<36,0$ & $<33,0$ \\
\hline Pontos outorgados & $35-40$ & $25<35$ & $15<25$ & \\
\hline Total de pontos do reprodutor & $86-100$ & $66<86$ & $40<66$ & $<40$ \\
\hline
\end{tabular}

Fonte: Fonseca et al. (1997).

\section{Resultados e Discussão}

As médias, os desvios-padrão, os coeficientes de variação e os valores mínimos e máximos para as variáveis estudadas estão apresentadas na Tabela 3. 
LOPES, F.G. et al. Utilização de dois métodos de classificação andrológica por pontos em touros jovens da raça Braford. PUBVET, Londrina, V. 7, N. 12, Ed. 235, Art. 1551, Junho, 2013.

Tabela 3. Características andrológicas dos touros jovens da raça Braford.

\begin{tabular}{lcccc}
\hline \multicolumn{1}{c}{ Características } & X \pm DP & CV & Mín. & Máx. \\
\hline Perímetro escrotal (cm) & $35,85 \pm 2,28$ & 6,36 & 30 & 41 \\
Turbilhão (0-5) & $4,01 \pm 0,84$ & 20,86 & 3 & 5 \\
Vigor (0-5) & $4,06 \pm 0,82$ & 20,21 & 0 & 5 \\
Motilidade (\%) & $80,29 \pm 8,31$ & 10,53 & 70 & 90 \\
Defeitos Maiores (\%) & $3,42 \pm 5,29$ & 154,48 & 0 & 30,0 \\
Defeitos Menores (\%) & $3,41 \pm 4,64$ & 136,05 & 0 & 28,0 \\
Defeitos Totais (\%) & $6,83 \pm 9,04$ & 132,35 & 0 & 42,0 \\
CAP (0-100) & $93,30 \pm 7,81$ & 8,37 & 58,5 & 100 \\
BSE (0-100) & $83,69 \pm 11,84$ & 14,15 & 42,5 & 100
\end{tabular}

Legenda: $\mathrm{X}=$ média; $\mathrm{DP}=$ Desvio padrão; $\mathrm{CV}=$ coeficiente de variação; Mín $=$ mínima; Máx. = Máxima.

Os resultados descritos neste estudo, se enquadram com os padrões recomendados por Fonseca et al. (1992) e pelo CBRA (1998).

$O$ valor médio observado para perímetro escrotal foi de $35,85 \pm 2,28 \mathrm{~cm}$. A média observada para turbilhonamento, vigor espermático e motilidade progressiva retilínea foi de 4,01 \pm 0,84, 4,06 $\pm 0,82$ e 80,29 $\pm 8,31 \%$, respectivamente.

Quanto às características morfológicas, foi observada média de 3,42 \pm $5,29,3,41 \pm 4,64,6,83 \pm 9,04 \%$, para os defeitos maiores, defeitos menores e defeitos espermáticos totais, respectivamente.

Apesar dos resultados obtidos, não foi possível realizar comparações com outros autores, haja vista a escassez de trabalhos sobre touros jovens da raça Braford, criados no Brasil.

Com relação aos dois métodos de classificação andrológica, o BSE e o CAP obtiveram pontuação média de $83,69 \pm 11,84$ (satisfatório) e 93,30 \pm 7,81 (excelente), respectivamente.

Neste caso, pode se observar que independente do método utilizado para 
LOPES, F.G. et al. Utilização de dois métodos de classificação andrológica por pontos em touros jovens da raça Braford. PUBVET, Londrina, V. 7, N. 12, Ed. 235, Art. 1551, Junho, 2013.

classificação andrológica, os touros foram considerados aptos à reprodução, com amplitude de idade jovem. Além disso, estes animais atingiram sua classificação máxima, de acordo com o método utilizado.

De acordo com Fonseca (1997), o estudo das associações entre as características seminais e perímetro escrotal é muito importante e, podem demonstrar, quais delas se correlacionam e se influenciam mutuamente.

Entre as características estudadas no presente trabalho, observou se correlação positiva entre o método CAP e o perímetro escrotal e com as características físicas do sêmen $(P<0.05)$.

Os valores obtidos nestas correlações foram $(r=0.310)$ entre CAP e perímetro escrotal, $(r=0.622)$ entre CAP e motilidade progressiva retilínea, $(r$ $=0.607$ ) entre CAP e vigor espermático. Já, para as características morfológicas do sêmen, as correlações foram consideradas negativas e $(P<0.05)$. Os valores obtidos foram $(r=-0.748)$ entre CAP e defeitos maiores e $(r=-0.758)$ entre CAP e defeitos espermáticos totais (Tabela 4).

Em estudos realizados com touros da raça Nelore, Salvador et al. (2002), Dias et al. (2006) e Folhadella et al. (2006) verificaram resultados semelhantes, com correlações positivas e significativas para CAP e perímetro escrotal e características físicas do sêmen e, negativas e significativas, para as características morfológicas do sêmen.

Quanto ao método BSE, pode se observar correlação positiva entre o perímetro escrotal e com as características físicas do sêmen e, negativas, para as características morfológicas do sêmen $(P<0.05)$.

Os valores obtidos nestas correlações foram $(r=0.711)$ entre BSE e perímetro escrotal, $(r=0.556)$ entre BSE e motilidade progressiva retilínea, $(r$ $=0.536)$ entre BSE e vigor espermático, $(r=-0.546)$ entre BSE e defeitos maiores e $(r=-0.538)$ entre BSE e defeitos espermáticos totais (Tabela 4).

Assim, com base nos resultados descritos, quanto maior a pontuação do perímetro escrotal maior a pontuação do método CAP ou do BSE, e quanto maior a pontuação da motilidade progressiva retilínea e vigor espermático, consequentemente, menores serão os valores para defeitos maiores e totais 
LOPES, F.G. et al. Utilização de dois métodos de classificação andrológica por pontos em touros jovens da raça Braford. PUBVET, Londrina, V. 7, N. 12, Ed. 235, Art. 1551, Junho, 2013.

espermáticos.

Outra abordagem do presente estudo foi correlacionar os dois métodos de classificação andrológica (BSE e CAP). Neste caso, pode se observar correlação positiva $(r=0.751)(P<0.05)$ (Tabela 4). Isto ocorreu, pelo fato de que os dois métodos de classificação andrológica, utilizam os mesmo parâmetro e valores para a formação do índice utilizando pesos diferentes na efetivação do valor final do índice.

Tabela 4. Correlação simples de Pearson entre dois métodos de classificação andrológica por pontos (CAP e BSE) e perímetro escrotal, motilidade progressiva retilínea, vigor espermático, defeitos maiores e totais espermáticos de touros jovens da raça Braford.

\begin{tabular}{ccccccc}
\hline Variável & \multicolumn{7}{c}{ Correlação } \\
\cline { 2 - 7 } & PE & Motilidade & Vigor & DM & DT & CAP \\
\cline { 2 - 7 } CAP & 0.310 & 0.622 & 0.607 & -0.748 & -0.758 & - \\
BSE & 0.711 & 0.556 & 0.536 & -0.546 & -0.538 & 0.751
\end{tabular}

Legenda: $\mathrm{PE}=$ perímetro escrotal; $\mathrm{DM}=$ defeitos maiores; $\mathrm{DT}=$ defeitos totais. $(\mathrm{P}<0.05)$.

\section{CONCLUSÕES}

Conclui-se que independente do método utilizado para classificação andrológica, os touros foram considerados aptos à reprodução, com amplitude de idade jovem. Além disso, atingiram sua classificação máxima, de acordo com o método utilizado.

\section{REFERÊNCIAS BIBLIOGRÁFICAS}

BLOM, E. Pathological condictions in the genital organs and in the semen of group for rejection of breeding bulls for import or export to and from Denmark, 1958-1982. Nordisk Veterinaer Medicin, v.35, n.3, p.105-130, 1983.

CHENOWETH, P. J.; BALL, L. Breeding soundness evaluation in bulls. In: MORROW, D. A. Current Therapy in Theriogenology. Saunders Company: Philadelphia, 1980, p.330-339.

COLÉGIO BRASILEIRO DE REPRODUÇÃO - Cbra. Manual para exame andrológico e avaliação de sêmen animal. 2.ed. Belo Horizonte: CBRA, 1998, p 49. 
DIAS, J.C.; ANDRADE, V.J.; FRIDRICH, A.B. et al. Estimativas de parâmetros genéticos de características reprodutivas de touros Nelore, de dois e três anos de idade. Arquivo Brasileiro de Medicina Veterinária e Zootecnia, v.58, n.3, p.388-393, 2006.

FOLHADELLA, I.M.; SÁ, W.F.; FERREIRA, A.M. et al. Características andrológicas de touros da raça Gir. Arquivo Brasileiro de Medicina Veterinária e Zootecnia, v.58, n.5, p.809-815, 2006.

FONSECA, V. O.; CRUDELI, G. A.; COSTA E SILVA, E. V. et al. Potencial reprodutivo de touros da raça Nelore (Bos taurus indicus) em monta natural: proporção touro:vaca 1:40 e fertilidade. Revista Brasileira de Reprodução Animal, v.15, n.1/2, p.103-108, 1991.

FONSECA, V.O.; SANTOS, N.R.; MALINSKI, P.R. Classificação andrológica de touros zebus (Bos taurus indicus) com base no perímetro escrotal e características morfo-físicas do sêmen. Revista Brasileira de Reprodução Animal, v.21, n.2, p.36-39, 1997.

FONSECA, V.O.; VALE FILHO, N.R.; MIES FILHO, A. ABREU, J.J. Procedimentos para exame andrológico e avaliação de sêmen animal. Belo Horizonte: Colégio Brasileiro de Reprodução Animal, 1992, 79 p.

MINITAB. Minitab for Windows - version 13.0. PA, USA: State College: Programa estatístico. 2000.

SALVADOR, D.F.; DIAS, J.C.; VALE FILHO, V.R et al. Perfil andrológico de touros da raça Nelore com três e quatro anos de idade, criados extensivamente em condições do estado do Mato Grosso do Sul. Revista Brasileira de Reprodução Animal, v.26, p.64-67, 2002

SILVA, A. L. M. Optimização do manejo reprodutivo de uma exploração de bovinos em regime extensivo. 2011. 74 f. Dissertação (Mestrado em medicina veterinária) - Universidade Lusófona de Humanidades e Tecnologias, Lisboa, 2011.

VALE FILHO, V. R. Andrologia no touro: avaliação genital, exame de sêmen e classificação por pontos. Revista Brasileira de Reprodução Animal, v.21, n.3, p.7-13, 1997.

VALE FILHO, V. R., QUIRINO, C. R.; ANDRADE, V. A. et al. Parâmetros genéticos da classificação andrológica por pontos (CAP), em touros da raça Nelore. Revista Brasileira de Reprodução Animal, v.23, p.253-255, 1999.

VALE FILHO, V. R.; ANDRADE, V. J.; QUIRINO, C. R. et al. Perfil andrológico de touros da raça Tabapuã (Bos taurus indicus) de um e dois anos de idade, criados extensivamente nos estados de Minas Gerais, Bahia e Espírito Santo, Brasil. Revista Brasileira de Reprodução Animal, v.25, n.2, p.189-192, 2001.

VASCONCELOS, C.P.; GUIMARÃES, J.D.; GUIMARÃES, S.E.F. et al. Estádio de maturidade sexual em touros da raça Nelore, dos 20 aos 22 meses de idade. Revista Brasileira de Reprodução Animal, v.27, n.2, p.174-176, 2003. 